London Cancer Network has devised and implemented a suspected cancer referral form for Primary Care Practitioners for patients to be seen within two weeks of referral to secondary care. One group referred on the suspected upper GI cancer referral form is patients unexplained iron deficiency anaemia (IDA) without other symptoms. Whilst IDA is a recognised finding in upper GI cancer we hypothesise that it is a rare presentation of upper GI cancer in the absence of other symptoms.

Methods The aim of this study is to assess the presenting symptoms in patients diagnosed with upper GI cancer when endoscopy is performed for anaemia as the primary indication.

A single centre, retrospective analysis of all patients undergoing endoscopy for IDA from August 2008 for 5 years at a District General Hospital in North London was performed. Data was collected using electronic patient records and unisoft endoscopy database. Those diagnosed with upper GI cancer were scrutinised for presence of symptoms in addition to anaemia at presentation.

Results Over the study period, 1529 patients were gastroscoped for IDA, and 1228 colonoscopied for IDA. 20 upper GI cancers (16 stomach, 4 oesophageal) were detected during the study. No patients with upper GI cancer had IDA alone with addition symptoms including weight loss (9 patients), malaena (3), dysphagia (3), abdominal pain (2), anorexia (2), abnormal CT scan (2), altered bowel habit (2). Other benign diagnosis at gastroscopy in anaemic patients included: Barrett's oesophagus (52), oesophagitis (159), oesophageal varices (11), gastric erosions (27), gastritis (438), pyloric stenosis (2), angiodysplasia (20), duodenal ulcer (35), duodenitis (139). In the group colonoscopied for anaemia findings included: Normal in 550,66 had colorectal cancer, polyps in 173, angiodysplasia in 33, and IBD in 16.

Conclusion From this study we conclude that upper GI cancer is diagnosed on gastroscopy in only $1.3 \%$ of patients presenting with IDA. When Upper GI cancer is diagnosis in IDA it is always associated with an additional symptom such as weight loss, anorexia, dysphagia, malaena or an abnormal CT scan.

Patients should not be referred with IDA on a suspected upper GI cancer referral form unless accompanied by additional alarm features. If a patient has isolated IDA and cancer is suspected a diagnostic colonoscopy is more rewarding than a gastroscopy and it is more appropriate to refer these patients to the colorectal cancer pathway. If similar findings are replicated than National guidelines should be informed and altered accordingly.

Disclosure of Interest None Declared.

\section{PTU-050 THE VALUE OF GASTROSCOPY IN THE INVESTIGATION OF NON CARDIAC CHEST PAIN}

SK Butt* ${ }^{*}$ K Besherdas. Gastroenterology, Chase Farm Hospital, London, UK

\subsection{6/gutjnl-2014-307263.124}

Introduction Non-cardiac chest pain (NCCP) is a common cause for referral to gastroenterology. Following the exclusion of a cardiac cause, NCCP is attributed to a variety of disorders, including gastroesophageal reflux disease (GORD), oesophageal dysmotility and oesophageal hypersensitivity, panic attack, musculoskeletal pain, and microvascular disease (cardiac syndrome $\mathrm{X})$. GORD is the most prevalent cause of NCCP, accounting for up to $60 \%$ of cases. The prevalence of GORD in NCCP has been studied by $\mathrm{pH}$ monitoring and found to be $41 \%$ to $43 \%$.
Methods The aim of this study was to assess the diagnostic yield of gastroscopy as a first line investigation in the management of patients with NCCP. A retrospective analysis from September 2000-March 2013 of the endoscopic findings (using the Endoscribe and unisoft endoscopy reporting system) along with patient case notes, of consecutive patients with NCCP undergoing gastroscopy, in a district general hospital in North London was undertaken.

Results A total of 146 patients (age range was 21 to 93 years with a male to female ratio of $68: 78)$ were identified as having had an upper GI endoscopy for chest pain alone. 49/146 (34\%) patients had a normal gastroscopy, 29/146 (20\%) had evidence of GORD (oesophagitis, peptic stricture, Barrett's oesophagus or hiatus, hernia), 27 patients (18\%) had gastritis, and 11 (7\%) with oesophageal motility disorders. Other findings included: 9 patients with duodenitis, 1 with gastric ulcer, 1 with oesophageal ulcer, 2 with gastric polyp.

Conclusion In this study, the diagnostic yield of gastroscopy as to a oesophageal cause of NCCP is poor. $73 \%$ of patients with NCCP had a normal or incidencal benign non-contributory findings at endoscopy. Only $27 \%$ of patients had evidence of GORD or oesophageal dysmotility. The majority of these could have been diagnosed by high dose acid suppression therapy trial. We conclude that endoscopy in patients presenting to gastroenterology with NCCP is not a cost effective diagnostic tool and would suggest other modalities such as high dose proton pump inhibitor trial, barium swallow or oesophageal manometry/pH testing be explored as first line investigations. Disclosure of Interest None Declared.

\section{PTU-051 ODYNOPHAGIA - A SYMPTOM WORTH ASKING ABOUT?}

S Subramaniam*, G Goodchild, K Besherdas. Department of Gastroenterology, Barnet and Chase Farm NHS Trust, London, UK

\subsection{6/gutjnl-2014-307263.125}

Introduction Odynophagia can be defined as a painful sensation in the oesophageal region that occurs in relation to swallowing. Unlike dysphagia, which has historically been an alarm symptom or warning sign of oesophageal cancer, odynophagia is not classified as an alarm symptom and does not form part of the suspected upper gastrointestinal (GI) cancer referral form in the UK. Endoscopy is the gold standard imaging modality for the diagnosis of mucosal lesions in the oesophagus. However, there is no clear data regarding the findings at endoscopy in patients scoped for odynophagia. Mucosal abnormalities even in the presence of typical symptoms of gastro-oesophageal reflux disease, namely heartburn and regurgitation are absent in up to $70 \%$. We hypothesise that the presence of odynophagia has a high predictive value of mucosal abnormality at endoscopy and aimed to assess the findings at endoscopy for patients scoped for odynophagia.

Methods A retrospective analysis of all patients who underwent upper GI endoscopy for odynophagia as a primary symptom over an 8-year period (2005-2013) within an NHS Trust in north London was performed. Data was obtained from the Unisoft Endoscopy reporting software. The findings at endoscopy in patients with odynophagia were scrutinised.

Results 50 patients were endoscoped for odynophagia during the study period. 34 of 50 patients (68\%) had oesophageal mucosal lesions (4 Barrett's mucosa, 2 candida oesophagitis, 14 reflux oesophagitis, 6 malignant tumour, 5 oesophageal stricture, 
3 oesophageal ulcers). 12\% (6 of 50) had oesophageal cancer. A further 10 had hiatus hernia, 1 had a motility disorder and 1 had oesophageal diverticulum.

Conclusion From this study, 68\% of patients endoscoped for odynophagia have a positive endoscopic mucosal abnormality. Odynophagia as a symptom has a high sensitivity for abnormal endoscopy. $12 \%$ of patients endoscoped for odynophagia had oesophageal cancer. This prevalence is similar to the diagnosis of cancer in patients referred on the 'two week wait upper GI cancer referral form'. We recommend the symptom of odynophagia be classified as an alarm symptom and those presenting with odynophagia all undergo upper GI endoscopy to define the exact mucosal abnormality and exclude oesophageal cancer.

Disclosure of Interest None Declared.

\section{PTU-052 DOES USE OF SEDATION AFFECT THE SPEED AT WHICH ENDOSCOPY IS PERFORMED AND NUMBER OF BIOPSIES OBTAINED IN BARRETT'S OESOPHAGUS?}

S Subramaniam*, H Defoe, A Chitembwe, J Ferrera, K Besherdas. Department of Gastroenterology, Barnet and Chase Farm NHS Trust, London, UK

\subsection{6/gutjnl-2014-307263.126}

Introduction Guidelines for Barrett's oesophagus (BE) screening recommend 2 yearly endoscopies with 4 quadrant biopsies every $2 \mathrm{~cm}$ for $\mathrm{BE}$ without dysplasia. There is increasing evidence that longer inspection time of $\mathrm{BE}$ segment is associated with increased detection of high-grade dysplasia and oesophageal cancer. In our experience, BE surveillance endoscopies have been undertaken both with and without sedation as no formal guidelines recommend use of one method over the other. Endoscopic procedures may be quicker in the unsedated patient and therefore these are likely to have lower Barrett's inspection time (BIT) and also fewer biopsies than in sedated patients. The aim of our study was to assess the prevalence of sedation use in $\mathrm{BE}$ surveillance endoscopy and to determine if this affected the time taken for the procedure and the number of biopsies obtained.

Methods A retrospective analysis of all patients who underwent surveillance endoscopy for BE over a 5 year period (2009-2013) in a large district general hospital in North London were identified using the audit tool on Unisoft Endoscopy reporting software. Data collection was done by endoscopy unit nursing staff. From each report, use of sedation, length of BE and number of biopsies taken from BE segment were recorded. The time taken for each BE endoscopy was also obtained from procedure logbooks. The mean length of time (LOT) per procedure was compared between sedated and unsedated endoscopies using a t test. A multiple linear regression fit was performed on the data using regressors sedation values, length of $\mathrm{BE}$ and number of biopsies taken.

Results 181 endoscopies for BE surveillance were performed over 5 years. 37 were excluded as insufficient data was available. Of the 144 endoscopies remaining, 73 were unsedated and 71 with sedation. The mean LOT for sedated compared with unsedated endoscopies was $12.47 \mathrm{~min}$ and $10.36 \mathrm{~min}$ respectively ( $\mathrm{p}$ $=0.05$, confidence interval $=-4.23,0.01)$. The average number of biopsies in sedated patients was 3.87 and 3.85 in the unsedated $(\mathrm{p}=0.47)$. The regression was a poor fit $\left(\mathrm{R}^{2}\right.$ adjusted $=$ $-0.00033)$ and the overall relationship not significant: $\mathrm{F}(2,141)$ $=0.976, \mathrm{p}=0.38$. $\mathrm{P}$ values for sedation $(\mathrm{p}=0.96)$ and length of $\mathrm{BO}(\mathrm{p}=0.16)$ did not achieve significance either.

Conclusion In our study of patients undergoing endoscopy for BE surveillance, the LOT of endoscopic procedure was greater in patients receiving sedation than unsedated patients. The length of $\mathrm{BE}$ or the use of sedation did not have a significant effect on the number of biopsies taken. Sedation use did not affect number of biopsies obtained and therefore may not increase dysplasia detection. We conclude that surveillance for BE patients can be performed without sedation.

Disclosure of Interest None Declared.

\section{PTU-053 IS IT WORTH REPEATING PREVIOUS UNREMARKABLE SB2 CAPSULES WITH THE NEW SB3?}

S Dunn*, R Bevan, L Neilson, R Keay, C Davison, F Butt, S Panter. South Tyneside NHS Foundation Trust, South Shields, UK

\subsection{6/gutjnl-2014-307263.127}

Introduction Small bowel capsule endoscopy (SBCE) has become a valuable tool for investigating the small bowel and technology is rapidly advancing. One of the most recent devices available for capsule endoscopy (Pillcam ${ }^{\circledR}$ SB3, Given Imaging) has improved image resolution and a variable frame rate. The aim of this work is to address whether these innovations lead to increased mucosal visualisation and diagnostic yield in clinical practice and therefore whether a repeat SB3 capsule should be considered in those patients with an equivocal SB2 result.

Methods A review was undertaken of the last 100 Pillcam ${ }^{\circledR}$ SB2 capsules and the first 55 Pillcam ${ }^{\circledR}$ SB3 capsules to be performed at South Tyneside District Hospital (14/01/13-12/12/13). Visualisation of the ampulla was used as a surrogate marker of mucosal visualisation and diagnostic yield was assessed by reviewing the reports. Statistical significance was calculated using Fisher's exact test.

Results Results are summarised in Table 1 below. The ampulla was visualised in 14\% of SB2 capsules and 18\% of SB3 capsules ( $p>0.05$ ). $44 \%$ of SB2 capsules were abnormal and SB3 capsules were abnormal in $62 \%$ of cases $(\mathrm{p}<0.05)$.

Conclusion It is recognised that the views obtained by SBCE can be compromised in the duodenum due to "rapid transit" and previous studies have suggested that due to this the ampulla of Vater is not often seen. ${ }^{1}$ Variable frame rates aim to address this by capturing more images when the capsule is moving quicker. We showed no statistically significant difference between ampullary visualisation of the SB2 and SB3 capsules, although the trend was to a higher percentage visualisation with the SB3 capsule. The overall yield of pathology from SB3 capsules was significantly higher than that in SB2 capsules. Given the overall increased yield of pathology it may be beneficial to repeat an SB3 capsule in someone with a previously equivocal SB2 result.

\begin{tabular}{llll}
\multicolumn{4}{l}{ Abstract PTU-053 Table 1} \\
\hline Capsule type & Number & Ampulla seen (\%) & Pathology found (\%) \\
\hline SB2 & 100 & $14(14 \%)$ & $44(44 \%)$ \\
SB3 & 55 & $10(18 \%)$ & $34(62 \%)$ \\
p value & & 0.495 & 0.044 \\
\hline
\end{tabular}

\section{REFERENCE}

1 Koulaouzidis A, Rondonotti E, Karargyris A. Small-bowel capsule endoscopy: A ten-point contemporary review. World Journal of Gastroenterology 2013;19 (24):3726-2840

Disclosure of Interest S. Dunn Grant/research support from: Aquilant Endoscopy, R. Bevan Grant/research support from: 